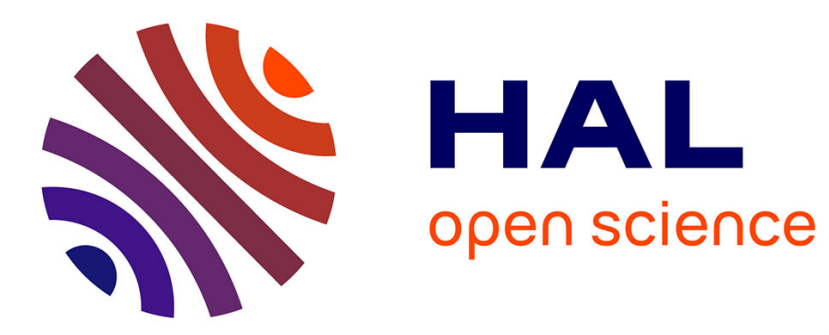

\title{
Exercise and bone mineral density in old subjects: theorical and practical implications
}

Thierry Paillard

\section{To cite this version:}

Thierry Paillard. Exercise and bone mineral density in old subjects: theorical and practical implications. Psychologie \& NeuroPsychiatrie du vieillissement, 2014, 12 (3), pp.267-273. 10.1684/pnv.2014.0491 . hal-02360979

\section{HAL Id: hal-02360979 \\ https://hal.science/hal-02360979}

Submitted on 13 Nov 2019

HAL is a multi-disciplinary open access archive for the deposit and dissemination of scientific research documents, whether they are published or not. The documents may come from teaching and research institutions in France or abroad, or from public or private research centers.
L'archive ouverte pluridisciplinaire HAL, est destinée au dépôt et à la diffusion de documents scientifiques de niveau recherche, publiés ou non, émanant des établissements d'enseignement et de recherche français ou étrangers, des laboratoires publics ou privés. 
Exercice et densité minérale osseuse chez les sujets âgés : implications théoriques et pratiques

Exercise and bone mineral density in old subjects: theorical and practical implications

Thierry Paillard

Laboratoire Activité Physique Performance et Santé (UPRES EA 4445), Université de Pau \& Pays de I'Adour, Département STAPS de Tarbes, ZA Bastillac Sud, 65000 Tarbes, France.

Thierry Paillard

Université de Pau et des Pays de l'Adour

Département STAPS

ZA Bastillac Sud

65000 TARBES

Tel : 0562566119

Fax : 0562566110

e-mail : thierry.paillard@univ-pau.fr 


\section{Résumé}

Avec l'avancée en âge, la diminution de la densité minérale osseuse est inéluctable. L'exercice physique régulier constitue un moyen physiologique susceptible d'atténuer les effets de la déminéralisation osseuse normale (i.e. non pathologique) particulièrement chez la personne âgée. En effet, l'exercice physique induit des contraintes mécaniques générant une déformation de l'os qui stimulent l'ostéogénèse et favorisent le remodelage osseux. La fonction ostéogénique des entraînements aérobies (e.g. marche, course) n'est effective que si l'intensité de l'exercice (i.e. les impacts au sol et donc les déformations osseuses) est de haute intensité et celle des entraînements de renforcement musculaire est effective que si les contractions musculaires effectuées sont dynamiques et de forte intensité. Les activités physiques réalisées en condition de décharge corporelle (e.g. natation, vélo) ou en condition statique (e.g. étirements, exercices d'équilibration) sont en revanche des activités parmi les moins ostéogéniques. La durée optimale d'un programme d'exercices significatif en termes de remodelage osseux se situe entre 4 et 6 mois. Les effets ostéogéniques de l'exercice régulier débute à partir de 2-3 séances hebdomadaires. L'activation de la fonction ostéogénique par le biais de l'exercice physique est plus difficile pour la femme vieillissante que pour l'homme vieillissant à cause de ses facteurs hormonaux moins favorables.

Mots clés : sujet âgé, exercice physique, densité minérale osseuse, volume d'activité physique, risque de fracture

\section{Points clés :}

- L'exercice physique dynamique (i.e. des activités physiques générant des pressions (impacts) mécaniques sur les os versus des activités n’en générant pas telles que celles réalisées en condition de décharge corporelle e.g. natation, vélo) régulier stimule l'activité des ostéoblastes et facilite l'activité des ostéocytes et l'absorption intestinale du calcium.

- Il impacterait négativement la production des ostéocytes en sclérostine dont la fonction est d'inhiber les ostéoblastes et (donc) la formation osseuse et induirait une augmentation de la production de facteurs anaboliques liée au métabolisme osseux. 
- Il augmenterait la concentration calcique de façon plus importante du côté concave que du côté convexe de la déformation osseuse induite par la pression mécanique ; d'où l'importance de varier la direction des contraintes mécaniques au cours de l'activité physique pour renforcer la résistance de l'os dans tous les plans.

- Plus l'intensité et la fréquence de l'exercice sont élevées, meilleure demeure la réponse ostéogénique tandis que la régularité de l'exercice représente un autre paramètre fondamental dans le maintien de la densité minérale osseuse.

- Chez la personne âgée, il existerait une relation entre le volume d'activité physique et la densité minérale osseuse (sur les sites anatomiques spécifiquement soumis à des contraintes mécaniques intenses et répétées) ainsi qu'une relation entre la densité minérale osseuse et le risque de fracture osseuse. 


\section{Abstract}

With age advancement, the decrease of bone mineral density is ineluctable. Physical exercise constitutes a physiological approach likely to attenuate or limit the effects of normal bone demineralization (i.e. not pathological) particularly in elderly subjects. Indeed, physical exercise induces mechanical constraints generating bone deformation which stimulates osteogenesis and favors bone remodelage. Physical activities achieved in condition of body discharge (e.g. swimming, cycling) or in static condition (e.g. stretching, balance) do not stimulate (or very weakly) osteogenesis. The osteogenic function of aerobic training (e.g. walking, running) is effective only if the intensity of exercise is high (i.e. the impacts on the ground and thus the bone deformation) and that of strength training is effective only if the completed muscular contractions are dynamic and carried out with heavy loads. The calcium concentration increase is greater on the concave side than on the convex side for the bones which undergo strong mechanical pressures during exercise. Hence, it is advisable to vary the directions of mechanical constraints during physical activity to strengthen the resistance of the bone in all the plans. In order to obtain significant effects in terms of bone remodelage, the optimal duration of training programs should last at least 4 to 6 months. The osteogenic effects of regular exercise begin from 2-3 weekly sessions. The activation of osteogenesis by means of physical exercise is more difficult in aging women than in aging men because of hormonal factors that are not favorable in aging women. At last, regular exercise is fundamental not only to maintain bone mineral density but also to reduce the risk of bone fracture since there is a relationship between the bone mineral density and the risk of bone fracture.

Key words: elderly subjects, physical exercise, bone mineral density, amount of physical activity, risk of fracture 
Avec l'avancée en âge, la diminution de la densité minérale osseuse est inéluctable. Elle se traduit par une diminution de la résistance mécanique de l'os à toute sorte de force (e.g. choc, pression, contrainte mécanique) qui s'exerce sur lui. Le risque de chute lié à l'altération de la fonction d'équilibration augmentant également sous l'effet de l'avancée en âge, le risque de fracture croît en conséquence avec l'âge [1].

L'exercice physique régulier constitue un moyen physiologique (avec l'alimentation) susceptible d'atténuer les effets de la déminéralisation osseuse normale (i.e. non pathologique) particulièrement chez la personne âgée. En effet, l'exercice physique induit des contraintes mécaniques générant une déformation de l'os qui stimulent l'ostéogénèse et favorisent le remodelage osseux chez les personnes vieillissantes [2].

A ce titre, les investigations observationnelles et interventionnelles ayant pour objectif d'étudier les effets préventifs de l'exercice régulier contre la déminéralisation osseuse ont été relativement conséquentes au cours des deux dernières décennies. Toutefois, même s'il est actuellement possible d'identifier les activités physiques favorables à l'ostéogenèse, la question des effets quantitatifs et qualitatifs de l'exercice considéré comme ostéogénique en termes de paramètres d'intensité, de durée et de fréquence sur la prévention de la déminéralisation osseuse chez les sujets âgés reste encore ouverte. C'est pourquoi l'objectif de cette courte revue consiste à présenter les principaux résultats des études traitant des effets préventifs des différents paramètres (cités ci-dessus) des exercices pratiqués chez la personne âgée contre l'altération de son appareil osseux.

\section{Vieillissement du tissu osseux}

La matière osseuse est un tissu vivant en perpétuel remaniement. Des cellules osseuses disparaissent et se créent quotidiennement. Deux voies antagonistes interagissent l'une sur l'autre (voie Wnt ou wingless-related integration site et voie RANK/RANKL/OPG ou 
receptor activator of nuclear factor-kappa B/receptor activator of nuclear factor-kappa $\mathrm{B}$ ligand/osteoprotegerin) pour assurer l'équilibre entre la formation et la résorption osseuses [3]. Ces deux voies régulent l'activité des ostéoblastes (responsables de la formation du tissu osseux) et des ostéoclastes (responsables de la résorption du tissu osseux).

Chez l'enfant et l'adolescent, la formation de la matière osseuse est excédentaire, c'est-à-dire que l'activité des ostéoblastes est supérieure à celle des ostéoclastes. Cet excédent permet la croissance osseuse. Chez le jeune adulte, les processus de résorption et de formation sont normalement étroitement liés de façon à ce que la quantité de résorption osseuse soit compensée par une quantité sensiblement égale de nouvelle formation osseuse; la masse osseuse régresse néanmoins légèrement et linéairement entre 20 et 50 ans. Ces processus sont régulés au niveau local par une multitude de cytokines et de facteurs de croissance [4]. En revanche, chez le sujet âgé, l'activité des ostéoclastes dépasse celle des ostéoblastes. Le nombre et l'activité des ostéoblastes diminuent et certaines trabécules individuelles disparaissent ou subissent une perforation [5]. Ceci engendre d'une part, une régression de l'espace nécessaire à la formation de nouvelles cellules osseuses, et d'autre part, une désorganisation du réseau trabéculaire. Ainsi, le vieillissement se caractérise par une réduction de la masse et un changement de l'architecture du tissu osseux. Ces modifications histologiques de l'os se définissent par le terme d'ostéopénie et sont également liées à un ralentissement de la vitesse du processus de remodelage osseux.

Selon l'organisation mondiale de la santé (OMS), une valeur de la densité minérale osseuse (DMO) comprise entre 1 et 2,5 déviations standard au-dessous de la densité normale moyenne des adultes jeunes correspond à l'ostéopénie et une valeur inférieure à 2,5 déviations standard correspond à l'ostéoporose. 
La prévalence de l'ostéoporose augmente avec l'avancée en âge. Les fractures ostéoporotiques affectent particulièrement l'extrémité proximale du fémur (e.g. le col du fémur), les corps vertébraux et l'extrémité distale du radius [6]. L'ostéoporose se manifeste après 40 ans et se montre plus sévère chez les femmes que chez les hommes surtout après la ménopause [7]. Après 65 ans, le risque fracturaire serait plus de 2 fois plus élevé chez la femme que chez l'homme [3, 6]. En revanche, les fractures de la hanche chez l'homme sont associés à une plus grande morbidité (et mortalité) que chez la femme [6,7]. Outre le facteur sexe, d'autres facteurs tels que l'inactivité physique, la déficience hormonale (en œstrogène chez la femme ménopausée), l'insuffisance nutritionnelle, les facteurs génétiques et le style de vie sont susceptibles d'accentuer ou d'atténuer le processus ostéoporotique [8]. A partir d'un certain seuil de déminéralisation osseuse, les os concernés par cet état biologique perdent de leur solidité et sont particulièrement exposés au risque fracturaire (Figure 1).

\section{Effets préventifs de l'exercice}

Le processus d'involution du tissu osseux peut être ralenti voire éventuellement inversé dans certaines circonstances très favorables chez les personnes âgées. Outre les conditions nutritionnelles et environnementales propices pour lutter contre ce processus, les sujets âgés doivent soumettre leur squelette à des contraintes mécaniques en pratiquant des exercices musculaires. En effet, les contraintes mécaniques induisant un certain seuil de déformation de l'os stimulent l'ostéogénèse et favorisent le remodelage osseux chez les personnes vieillissantes [2]. L'exercice physique stimule l'activité des ostéoblastes et facilite l'activité des ostéocytes [9] et l'absorption intestinale du calcium [8]. Il impacterait négativement la production des ostéocytes en sclérostine dont la fonction est d'inhiber les ostéoblastes et (donc) la formation osseuse et induirait une augmentation de la production de facteurs anaboliques liée au métabolisme osseux [10]. L'activité physique agirait favorablement sur la masse et l'architecture osseuses chez la personne âgée. 
Cependant, les effets de l'activité physique sur la DMO (exprimée en g/cm2) ne sont pas univoques. Pour certains auteurs, un programme d'activité physique peut retarder ou même inverser le processus de déminéralisation osseuse. Pour d'autres auteurs, les effets d'un programme d'entraînement seraient moins significatifs sur la DMO. En fait, le processus de minéralisation osseuse peut être plus ou moins influencé par la nature, l'intensité, la durée et la fréquence des activités physiques entreprises. Chez les sujets âgés, le facteur sexe peut également influencer les capacités d'adaptation de leurs appareils osseux sous l'effet de l'exercice musculaire.

Le processus ostéogénique dépend en partie de la nature de l'activité physique pratiquée et de l'impact des contraintes mécaniques qu'elle induit sur le squelette. Les entraînements aérobies de haute intensité et le travail de renforcement musculaire sont de nature à activer l'ostéogénèse. Un entraînement aérobie de 18 mois composé de marche, de montées d'escalier, d'efforts sur ergocycle et de jogging à raison de 3 fois par semaine, pendant 50 minutes, à intensité soutenue (55 à 75\% du VO2 max), favorise le maintien de la DMO au niveau du col du fémur chez des femmes ménopausées âgées de 52-53 ans [11]. Globalement, les activités qui impliquent les plus fortes contraintes mécaniques sont celles qui engendrent les effets positifs les plus importants sur le tissu osseux. En effet, des activités comme la danse (rythmique), la gymnastique (dynamique) et le step qui impliquent la réalisation d'impulsions et de réceptions corporelles générant des impacts au sol importants, sont des activités parmi les plus ostéogéniques susceptibles d'être pratiquées par les personnes âgées alors que des activités comme la natation et le vélo qui se pratiquent en condition de décharge corporelle n'engendrant pas d'impacts au sol sont des activités parmi les moins ostéogéniques. Enfin, pour une activité physique donnée telle que le renforcement musculaire au pouvoir ostéogénique important, certains mouvements sont davantage ostéogéniques que 
d'autres. Les actions musculaires de tirage seraient plus efficaces que les actions de portées car elles entraînent une déformation (contrainte mécanique) osseuse plus importante [8].

La nature des contraintes mécaniques demeure fondamentale au processus de minéralisation osseuse. Les contraintes dynamiques sont davantage ostéogéniques que les contraintes statiques. En outre, l'orientation des contraintes est également déterminante. L'idéal étant de pratiquer une activité physique dynamique exerçant des pressions mécaniques variées dans leurs directions sur un grand nombre de segments. La concentration calcique est plus importante du côté concave que du côté convexe de la déformation osseuse induite par la pression mécanique [12]. D'où l'importance de varier la direction des contraintes mécaniques au cours de l'activité physique pour renforcer la résistance de l'os dans les 3 plans (quand c'est possible !).

Une méta analyse de Layne et Nelson [13] a fait le point sur l'optimisation de l'intensité de l'entraînement à prescrire afin de favoriser la minéralisation osseuse. Cette étude atteste qu'une grande partie des travaux effectués sur ce thème abonde dans le sens où un programme de renforcement musculaire de haute intensité agit de façon bénéfique sur la DMO. Taaffe et al. [14] ont effectué une étude d'une durée de 52 semaines chez des femmes âgées de 65 à 69 ans pratiquant 3 fois par semaine des exercices de renforcement musculaire. Ils ont constitué 3 groupes, dont un a servi de groupe témoin. Les deux autres groupes ont réalisé des programmes de musculation de haute ( $80 \% 1 \mathrm{RM}$ i.e. répétition maximale) ou de basse intensité (40\% $1 \mathrm{RM})$. Seul, le groupe ayant pratiqué à une haute intensité a pu maintenir sa valeur initiale de DMO au niveau des fémurs. Le groupe témoin et le groupe ayant pratiqué à $40 \%$ d'intensité ont vu leur DMO régresser. Par ailleurs, selon Pruitt et al. [15], ce même programme n'engendre pas de différence de DMO entre les 3 groupes au niveau de la colonne vertébrale. A partir de là, la DMO se stabiliserait seulement sur les sites osseux particulièrement stimulés par l'exercice musculaire. Deux revues de la littérature concluent 
que le renforcement musculaire de haute intensité peut même augmenter la DMO sur les sites anatomiques qui subissent les plus fortes pressions mécaniques chez des femmes ménopausées [16, 17]. Une activité physique stimulant particulièrement les quadriceps engendre une augmentation de la DMO du col du fémur, alors qu'une stimulation de l'iliopsoas génère une élévation de la DMO de la colonne vertébrale [12].

La durée d'un programme d'entraînement influe sur le processus de minéralisation osseuse. Un programme de renforcement musculaire relativement intense (monter 300 marches d'escaliers par séance, 4 séances hebdomadaires) d'une durée de 6 semaines chez des femmes âgées de 62 à 75 ans n'a pas suffi à augmenter la DMO du col du fémur et de la colonne lombaire (L1-L4) [18]. La durée optimale d'un programme d'exercices significatif en termes de remodelage osseux se situe entre 4 et 6 mois [19]. Cependant, une durée plus longue ne constitue pas un gage d'amélioration systématique puisqu'un programme de musculation de forte intensité étalé sur deux ans (2 périodes de 42 semaines séparées par 10 semaines de repos et de tests), n'a pas induit d'augmentation de la DMO de la colonne lombaire et du corps entier chez les 57 sujets du groupe expérimental par rapport aux 56 sujets du groupe contrôle (tous âgés entre 60 et 80 ans) [20].

La fréquence des séances hebdomadaires d'activité physique demeure également un critère déterminant pour lutter efficacement contre le processus de déminéralisation osseuse. Deux séances d'activité physique hebdomadaires impliquant de fortes contraintes mécaniques semblent être le minimum pour favoriser la conservation de la DMO chez le sujet âgé. Dans une optique d'augmentation de la DMO, une fréquence supérieure paraît nécessaire mais ne constitue toutefois pas une garantie de résultat positif selon le statut physiologique et les conditions nutritionnelles et environnementales de l'individu. 
Chez des sujets âgés fragiles présentant de faibles capacités d'équilibration et de force musculaire ainsi qu'une faible DMO, le risque de chute et/ou de fractures spontanées demeure important. Pour ce type de sujets, le dilemme consiste à proposer une activité susceptible d'améliorer les paramètres physiques et physiologiques précédemment déclinés tout en évitant de mettre en péril leur intégrité physique à travers une activité physique stimulante et dynamique. La technique de vibration entière du corps (le sujet placé en position érigée sur une plate-forme vibrante) constitue à ce titre une certaine opportunité. En effet, certaines études montrent que la pratique régulière de la vibration améliore la DMO (ainsi que la force musculaire et l'équilibre postural) [e.g. 21, 22]. Toutefois, les conclusions d'une très récente méta-analyse [23] divergent notablement à ce propos. Leung et al. [23] suggèrent que les différents paramètres de stimulation (e.g. fréquence de vibration corporelle, inclinaison de la forme-forme vibrante, durée des séances, nombre de séances hebdomadaires, durée du programme, sites anatomiques osseux évalués) peuvent influer sur les résultats. Ainsi, d'autres travaux liés à la technique de vibration corporelle faisant varier ces paramètres méritent d'être effectués pour maitriser davantage ses effets sur la fonction ostéogénique des sujets âgés.

Par ailleurs, le facteur sexe influence également les effets induits par l'activité physique sur la DMO. En effet, une activité comme la marche qui induit certaines contraintes mécaniques et demeure donc relativement ostéogénique, peut s'avérer inefficace pour lutter contre la diminution de la DMO chez les femmes ménopausées [17, 24]. En revanche, la pratique de la marche n'engendre certes, pas d'augmentation de la DMO chez des sujets masculins ayant sensiblement le même âge mais elle la stabilise [25]. Les contraintes mécaniques même fréquentes induites par la marche sont probablement insuffisantes pour amplifier le processus de minéralisation osseuse chez les sujets âgés tandis qu'elles peuvent vraisemblablement, au mieux, stabiliser la DMO surtout chez les femmes âgées. Seule la pratique de la marche 
intense et pratiquée à un rythme très soutenu aurait un rôle ostéogénique significatif. Hatori et al. [26] ont en effet comparé 3 groupes composés de femmes ménopausées, deux ont réalisé un programme de marche (30 minutes, 3 fois par semaine, pendant 7 mois) dont un à une intensité correspondant à une valeur de fréquence cardiaque supérieure au seuil anaérobie $(n=12)$ et l'autre à une intensité inférieure à ce seuil $(n=9)$, le troisième était le groupe contrôle $(n=12)$. Seul le groupe ayant pratiqué à une intensité supérieure au seuil anaérobie a élevé sa DMO lombaire $(+1,1 \%)$ évaluée par absorptiométrie biphotonique à rayon $\mathrm{X}$ par comparaison au groupe ayant pratiqué à une intensité inférieure au seuil anaérobie $(-1,0 \%)$ et au groupe contrôle $(-1,7 \%)$ dont la DMO lombaire a diminué [26]. Par ailleurs, dans le cadre d'un programme d'activités physiques aérobies combinées (ergocycle, marche, stretching) de 14 mois chez des sujets âgés de plus de 60 ans, la DMO a augmenté chez les hommes mais pas chez les femmes [27]. Globalement, il est probable que l'exercice physique chez les femmes ménopausées puisse ralentir ou stabiliser le processus de déminéralisation osseuse mais pas l'inverser ou seulement dans le cadre d'activités fortement ostéogéniques (à impacts mécaniques élevés). En effet, dans le cadre d'un programme de renforcement musculaire, des femmes âgées $(n=20)$ soumises à 2 entraînements hebdomadaires pendant 1 an des muscles ilio-psoas, rotateurs latéraux (ou pelvi-trochantériens) et érecteurs du rachis lombaire ont vu leur DMO fémorale et lombaire s'accroître de $0,9 \%$ et $1,0 \%$ tandis que les femmes du groupe témoin $(n=19)$ de ce protocole expérimental ont vu leur DMO diminuer de $-2,5 \%$ et $-1,8 \%$ respectivement ( $\mathrm{p}=0,02$ et $\mathrm{p}=0,04$ respectivement) [28].

Lorsque les conditions environnementales, nutritionnelles et physiologiques sont favorables, les activités de renforcement musculaire intenses pourraient augmenter modérément la DMO chez les sujets âgés, tandis que les activités de type endurance (e.g. la marche) pratiquées isolément ne peuvent probablement que la maintenir (ou l'augmenter faiblement). Les activités pratiquées en décharge corporelle (vélo, natation...) s'avèrent, quant à elles, 
manifestement inopérantes dans une optique d'augmentation ou même de stabilisation de la DMO. Par ailleurs, l'efficacité d'un exercice physique relève plus de son intensité et de sa fréquence que de sa durée.

\section{Risque de fracture}

Le taux d'incidence de fractures de hanches et de fractures vertébrales demeure plus faible chez des sujets âgés physiquement actifs comparativement à des sujets âgés non actifs $[29,30$, 31]. Le suivi de 9704 femmes de plus de 65 ans durant environ 8 ans (7,6 années) indique que les personnes appartenant aux quintile et quartile les plus élevés en termes de niveau (volume) d'activité physique présentent un taux de fractures de hanche $36 \%(\mathrm{RR}=0.64$; IC 95\% [0.450.891]) plus faible que celles appartenant au quintile le plus bas [32]. Ces auteurs précisent que les femmes actives qui pratiquent à une intensité allant de modérée à vigoureuse diminuent de $42 \%$ et $33 \%$ le risque de fracture de hanche et de vertèbre respectivement en comparaison avec les femmes inactives. Dans le même sens, il existerait une relation entre la DMO et le risque fracturaire ostéoporotique $[33,34,35]$. Néanmoins, outre la valeur de la DMO, d'autres facteurs environnementaux et génétiques ainsi que la probabilité de chute accentuent ou atténuent ce risque fracturaire [36]. Le temps de maintien en appui monopodal demeure particulièrement discriminant en termes de risque fracturaire du radius chez les sujets de plus de 65 ans, particulièrement s'il est inférieur à 15 secondes [33]. En pratique, à titre préventif, il conviendrait de soumettre les sites particulièrement fragiles au regard du risque fracturaire à des contraintes mécaniques fréquentes et relativement intenses avec des activités particulièrement adaptées au statut physiologique de l'individu concerné. Il s'avère par ailleurs que plus un individu est actif, plus son risque de chute et donc de fracture augmente, surtout s'il pratique en milieu naturel comportant certains risques aggravants (e.g. terrain glissant, bosselé). 


\section{Conclusion}

Chez le sujet âgé, il existerait une relation entre son volume d'activité physique et sa DMO (sur les sites anatomiques spécifiquement soumis à des contraintes mécaniques intenses et répétées) ainsi qu'une une relation entre sa DMO et son risque de fracture osseuse. Dans une optique de maintien de la DMO, la régularité de l'exercice demeure fondamentale (durant toute la vie). Plus l'intensité de l'exercice est élevée meilleure demeure la réponse ostéogénique. Une fréquence d'au moins 2-3 séances d'activité physique hebdomadaires est nécessaire pour optimiser les effets préventifs sur la déminéralisation osseuse. 


\section{Références}

1. Nitz JC, Stock L, Khan A. Health-related predictors of falls and fractures in women over 40. Osteoporos Int 2013; 24: 613-21.

2. Srinivasan S, Gross TS, Bain SD. Bone mechanotransduction may require augmentation in order to strengthen the senescent skeleton. Ageing Res Rev 2012; 11: 353-60.

3. Mitchell BD, Streeten EA. Clinical impact of recent genetic discoveries in osteoporosis. Appl Clin Genet 2013; 6:75-85.

4. American Geriatrics Society (Fondation IPSEN, Société Française de Gérontologie, IPSEN). Syllabus Geriatrics. Paris: Auto-édition, Allard/Ipsen, 1998.

5. Marie PJ, Kassem M. Extrinsic mechanisms involved in age-related defective bone formation. J Clin Endocrinol Metab 2011; 96: 600-9.

6. Khosla S, Amin S, Orwoll E. Osteoporosis in men. Endocr Rev 2008; 29: 441-64.

7. Guggenbuhl P. Osteoporosis in males and females: Is there really a difference? Joint Bone Spine 2009; 76: 595-601.

8. Stransky M, Rysava L. Nutrition as prevention and treatment of osteoporosis. Physiol Res 2009; 58 (Suppl 1): 7-11.

9. Ozcivici E, Luu YK, Adler B, Qin YX, Rubin J, Judex S, et al. Mechanical signals as anabolic agents in bone. Nat Rev Rheumatol 2010; 6: 50-9.

10. Bosković K, Gava BP, Grajić M, Madić D, Obradović B, Todorović ST. Adapted physical activity in the prevention and therapy of osteoporosis. Med Pregl 2013 (Abstract); 66: 221-4.

11. Heinonen A, Oja P, Sievanen H, Pasanen M, Vuori I. Effect of two training regimens on bone mineral density in healthy perimenopausal women: a randomized controlled trial. $J$ Bone Miner Res 1998; 13: 483-90.

12. Paillard T. Vieillissement et Condition Physique. Paris : Edition Ellipses, 2009.

13. Layne JE, Nelson ME. The effects of progressive resistance training on bone density: a review. Med Sci Sports Exerc 1999; 31: 25-30.

14. Taaffe DR, Pruitt L, Pyka G, Guido D, Marcus R. Comparative effects of high- and lowintensity resistance training on thigh muscle strength, fiber area, and tissue composition in elderly women. Clin Physiol 1996; 16: 381-92.

15. Pruitt LA, Taaffe DR, Marcus R. Effects of a one - year high - intensity versus low intensity resistance training program on bone mineral density in older women. $J$ Bone Miner Res 1995; 10: 1788-95.

16. Zehnacker $\mathrm{CH}$, Bemis-Dougherty A. Effect of weighted exercises on bone mineral density in post menopausal women. A systematic review. J Geriatr Phys Ther 2007; 30: 79-88.

17. Gutin B, Kasper MJ. Can vigourous exercise play a role in osteoporosis prevention. A review. Osteoporos Int 1992; 2: 55-69. 
18. Paillard T, Lafont C, Costes-Salon MC, Vellas B, Dupui P. Comparison between three strength development methods on body composition in healthy elderly women. J Nutr Health Aging 2003; 7: 117-9.

19. Bonaiuti D. The conclusions of the systematic revision with the results from the major clinical and experimental studies. Eura Medicophys 2004; 40: 239-46.

20. McCartney N, Hicks Al, Martin J, Webber CE. A longitudinal trial of weight training in the elderly: continual improvements in years 2. J Gerontol A Biol Sci Med Sci 1996; 51: 42533.

21. Pang MY. Whole body vibration therapy in fracture prevention among adults with chronic disease. World J Orthop 2010; 1: 20-5.

22. Zha DS, Zhu QA, Pei WW, Zheng JC, Wu SH, Xu ZX, et al. Does whole-body vibration with alternative tilting increase bone mineral density and change bone metabolism in senior people? Aging Clin Exp Res 2012; 24: 28-36.

23. Leung KS1, Li CY, Tse YK, Choy TK, Leung PC, Hung VW, Chan SY, Leung AH, Cheung WH. Effects of 18-month low-magnitude high-frequency vibration on fall rate and fracture risks in 710 community elderly--a cluster-randomized controlled trial. Osteoporos Int $2014 ; 25: 1785-95$.

24. Kerr D, Morton A, Dick I, Prince R. Exercise effects on bone mass in postmenopausal women are site-specific and load-dependent. J Bone Miner Res 1996; 11: 218-25.

25. Paillard T, Lafont C, Costes-Salon MC, Rivière D, Dupui P. Effects of brisk walking on postural balance, locomotion, body composition and aerobic capacity in healthy ageing men. Int J Sports Med 2004; 25: 539-46.

26. Hatori M, Hasegawa A, Adachi H, Shinozaki A, Hayashi R, Okano H, et al. The effects of walking at the anaerobic threshold level on vertebral bone loss in postmenoposal women. Calcif Tissue Int 1993; 52: 411-4.

27. Blumenthal JA, Emery CF, Madden DJ, Schniebolk S, Riddle MW, Cobb FR, et al. Effects of exercise training on bone density in older men and women. J Am Geriatr Soc 1991; 39: $1065-70$

28. Nelson ME, Fiatarone MA, Morganti CM, Trice I, Greeberg RA, Evans WJ. Effects of high- intensity strength training on multiple risk factors for osteoporotic fractures. A randomized controlled trial. JAMA 1994; 272: 1909-14.

29. Marks R, Allegrante JP, Ronald MacKenzie C, Lane JM. Hip fractures among the elderly: causes, consequences and control. Ageing Res Rev 2003; 2: 57-93.

30. Sinaki M, Itoi E, Wahner HW, Wollan P, Gelzcer R, Mullan BP, et al. Stronger back muscles reduce the incidence of vertebral fractures: a prospective 10 year follow-up of postmenopausal women. Bone 2002; 30: 836-41.

31. Cumming RG, Nevitt MC, Cummings SR. Epidemiology of hip fractures. Epidemiol Rev 1997; 19: 244-57. 
32. Gregg EW, Cauley JA, Seeley DG, Ensrud KE, Bauer DC. Physical activity and osteoporotic fracture risk in older women. Study of osteoporotic fractures research group. Ann Intern Med 1998; 129: 81-8.

33. Sakai A, Oshige T, Zenke Y, Yamanaka Y, Otsuka H, Nakamura T. Shorter unipedal standing time and lower bone mineral density in women with distal radius fractures. Osteoporos Int 2010; 21: 733-9.

34. Lane NE. Epidemiology, etiology, and diagnosis of osteoporosis. Am J Obstet Gynecol 2006; 194 (2 Suppl): 3-11.

35. Dargent-Molina P, Douchin MN, Cormier C, Meunier PJ, Bréart G. Use of clinical risk factors in elderly women with low bone mineral density to identify women at higher risk of hip fracture: The EPIDOS prospective study. Osteoporos Int 2002; 13: 593-9.

36. Kaptoge S, Benevolenskaya LI, Bhalla AK, Cannata JB, Boonen S, Falch JA, et al. Low BMD is less predictive than reported falls for future limb fractures in women across Europe: results from the European Prospective Osteoporosis Study. Bone 2005; 36: 387-98. 


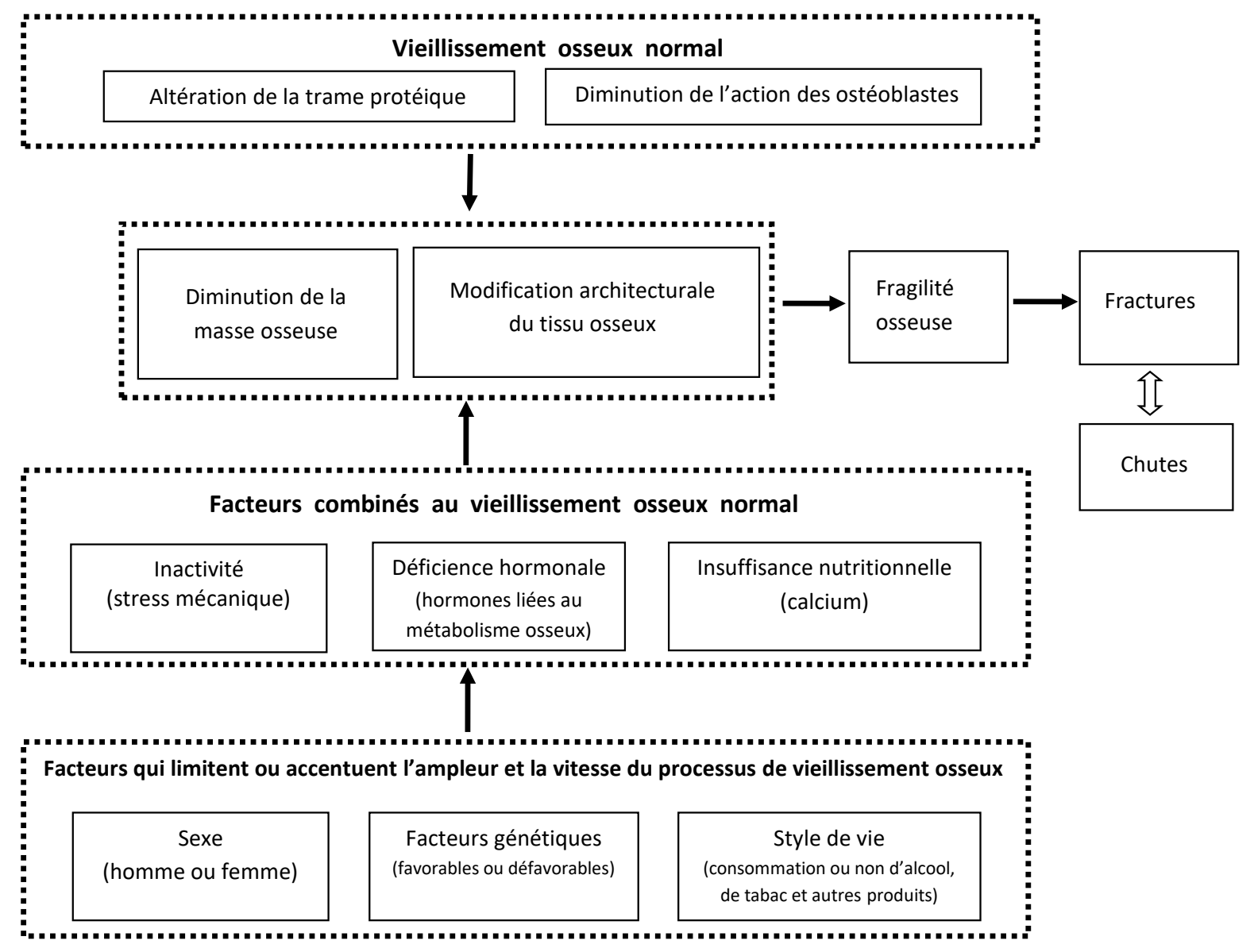

Figure: Représentation schématique du processus de vieillissement osseux et de ses conséquences (adaptée de Paillard [12]).

Figure: Schematic representation of the process of bone alteration related to age and its consequences. 